\title{
Bone tuberculosis: a case report on child
}

\author{
Fabiana Cristina Fulco Santos ${ }^{[1]}$, André Luiz Alves do Nascimento ${ }^{[1]}$, \\ Laís Ariane de Siqueira Lira ${ }^{[1]}$, Juliana Figueirêdo da Costa Lima ${ }^{[1]}$, \\ Rosana de Albuquerque Montenegro ${ }^{[1]}$, Lilian Maria Lapa Montenegro ${ }^{[1]}$ \\ and Haiana Charifker Schindler ${ }^{[1]}$
}

[1]. Laboratório de Imunoepidemilogia, Departamento de Imunologia, Centro de Pesquisas Aggeu Magalhães, Fundação Oswaldo Cruz, Recife, PE.

\begin{abstract}
The authors report a case of a 12-year-old child with a complaint of pain and deformity in the lower thoracic region that had lasted for two years. Clinical, epidemiological and laboratory characteristics associated with images of apparent damage in the T9-T10 and T11-T12 vertebrae taken by radiography of the thoracic spine and nuclear magnetic resonance in addition to the positivity of the molecular test based on the polymerase chain reaction, led to tuberculous spondylitis being diagnosed and specific therapy was started. Culture of vertebral biopsy was positive for Mycobacterium tuberculosis after thirty days.
\end{abstract}

Keywords: Tuberculosis. Spondylitis. PCR.

\section{INTRODUCTION}

Bone tuberculosis (TB) is one of the forms of extrapulmonary tuberculosis that affects, above all, the spine, long bones and joints. It is estimated that about 76,000 cases of TB were reported in Brazil in 2010, of which 441 were related to bone TB ${ }^{1}$.

Tuberculous spondylitis is the most common form of extrapulmonary TB which is around $50 \%$ of cases. This refers to the infection of one or more vertebral bodies by Mycobacterium tuberculosis with or without involvement of the spinal canal ${ }^{2,3}$. It occurs as a result of the hematogenous spread or erosion of adjacent caseating lymphnodes after a primary pulmonary infection that is not generally evident in children as they are more vulnerable to the occurrence of discitis because of the persistence of the anastomosis between the motor endplate and the spinal disc ${ }^{4}$.

Detecting M. tuberculosis in the initial phase of the disease is crucial to an early start to treatment must be made. Diagnosis is becomes difficult in uncommon extrapulmonary forms such as neurotuberculosis, renal and osteoarticular TB. This is true especially in children, once there are few bacilli in those individuals. Besides, obtaining extrapulmonary material generally requires invasive procedures and hospital care 5

New techniques based on molecular methods have been developed to aid the diagnosis of paucibacillary TB. In this regard single-tube nested PCR (STNPCR) is faster and presents

\footnotetext{
Address to: Dra. Haiana Charifker Schindler. Lab. Imunoepidemiologia/ Depto. Imunologia/CPqAM/FIOCRUZ. Av. Prof Moraes Rêgo s/n, Cidade Universitária, 50670-420 Recife, PE, Brasil.

Phone: 5581 2101-2569; 5581 9161-1333

e-mail: haia@cpqam.fiocruz.br

Received 20 September 2012

Accepted 15 February 2013
}

a minor contamination compared to conventional polymerase chain reaction (PCR) techniques. SNTPCR has shown high sensitivity and specificity in detecting the Koch's bacillus ${ }^{6}$.

The objective of this report is to describe a child case of spondylitis on the lower thoracic spine. And thus show the importance of identifying the disease as soon as possible, because it is a rare and severe condition that can leave to serious sequelae.

\section{CASE REPORT}

The patient was a 12-year-old Brazilian female school pupil, with darkish skin, from Taquaritinga do Norte (Northeast of Brazil, Pernambuco). This child related pain in her lower thoracic spine for 2 years. During this period she had used the health service in her town on several occasions, and had been treated with analgesics and/or non-hormonal, anti-inflammatory drugs.

As the patient showed no clinical evolution and there was an increase in the frequency and intensity of pain associated with difficulty in locomotion and a deformity in the lower thoracic region, she went to the orthopedics clinic at the Hospital das Clinicas/UFPE. She made a complete blood cell $(\mathrm{CBC})$ test to include the erythrocyte sedimentation rate, chest and (posteroanterior and lateral) thoracolumbar spine X-rays, nuclear magnetic resonance (NMR) and a biopsy of the site. The patient was included in the research protocol of the Immunoepidemiology Laboratory, CPqAM/FIOCRUZ so that the molecular test (STNPCR) could be conducted on peripheral blood, urine and bone aspirate of the T9 and T10 vertebrae.

As personal antecedents, at first, there was no history of contact with an adult with tuberculosis (although at the end of treatment, an adult with TB at her school was identified), a non-reactor Mantoux test and the presence of the scar from the BCG vaccination.

At the physical examination, she had showed good general state of health, with o fever, red-cheeked, weighed of 
$38.2 \mathrm{~kg}$ ( $\mathrm{p}>95$ - National Center for Health Statistics), and blood pressure of $120 / 80 \mathrm{mmHg}$. Heart and lung auscultation were normal with no hepatosplenomegaly or adenomegalies. She was observed to have postural alteration, with pain in the thoracolumbar with a kyphosis formation at the level of the lower thoracic spine, a limping gait, inability to flex the lumbodorsal spine and pain with palpation of the paravertebral fossas. No other external signs of inflammation were detected, with sensitivity and muscle strength symmetrically preserved in the lower limbs.

The $\mathrm{CBC}$ showed an increase in the erythrocyte sedimentation rate. The chest X-ray was normal and the spine showed thoracolumbar kyphosis, rarefaction of the vertebral bodies and collapse of the T11 and T12 vertebrae, with a reduction in the corresponding distal space.

The NMR of the thoracic spine showed signal alteration at T9, T10, T11 and T12 vertebral bodies with post-contrast enhancement, a slightly increase of prevertebral soft tissues and collapses of the T11-T12 vertebral bodies with their posterior displacement. Those alterations determining compression on the ventral face of the medulla and the appearance of kyphosis in this region. Despite the compressive effect exerted by the posterior displacement of the T-11 and T-12 vertebral bodies, the spinal cord maintained a normal signal intensity (Figures $\mathbf{1}$ and $\mathbf{2}$ ).

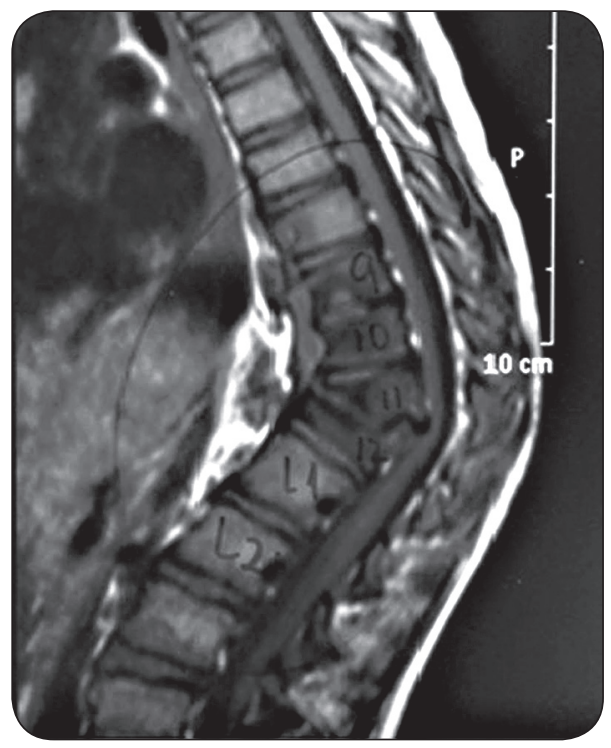

FIGURE 1 - Sagittal magnetic resonance giving evidence of the collapse of the T11-T12 vertebral bodies with their posterior displacement, determining compression on the ventral surface of the medulla and the appearance of kyphosis of the thoracic spine in this region.

Histological examinations revealed bone fragments of the spine affected by the chronic inflammatory process in addition to extensive fibrosis and nonspecific lymphoplasm infiltrate. The assay for alcohol-acid resistant bacilli, fungi and pyogenic bacteria produced negative results. Culture of the aspirate of the affected vertebrae revealed the growth of $M$. tuberculosis after 30 days of seeding.

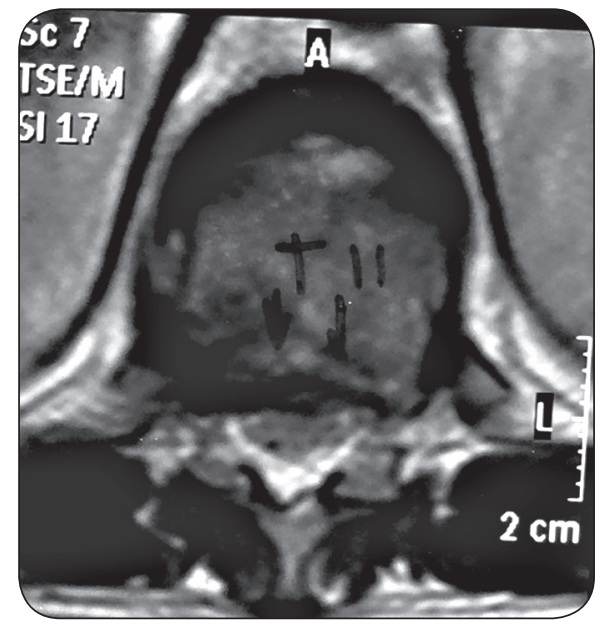

FIGURE 2 - Axil magnetic resonance, noting that the destruction of the T11 vertebral body and the reduction in the amplitude of the oblongata determines compression on the ventral surface of the medulla.

Each biological sample, after DNA extraction, was subjected to the molecular STNPCR technique using IS6110 primer as the protocol described by Lima et al. The specimens of bone aspirate and urine were positive for the M. tuberculosis complex.

The specific scheme for treating bone tuberculosis as recommended by the Ministry of Health of Brazil, was started in 2009. As surgery was not recommended, a plaster cast was put in place to give greater stability to the spine.

After three months of treatment the child had a decrease in symptoms with an obvious clinical improvement. Despite having evolved well in the aspect of control the infection, she remained with thoracic kyphosis.

\section{DIscussion}

Osteoarticular TB currently accounts for $35 \%$ of all cases of the extrapulmonary form of the disease, which is more commonly found in children and the in elderly. In vertebrae, the segment most often affected is the thoracic spine ${ }^{7}$. In this case study, the patient had residual kyphosis in the thoracic spine related to the collapse of the T11-T12 vertebral bodies.

Possibly, the low specificity of the clinical condition and the limited suspicion with regard to TB as the cause of osteoarticular pain were responsible for the delay in diagnosis. This delay also favors the occurrence of residual deformity, since the destruction of bone is slow and progressive.

Sarah et al., in a retrospective study of 21 patients with tuberculous spondylitis, with an average age of 9.7 years old, from an endemic area and with diagnosis in up to four months, verified that back pain is the most common symptom. Microbiological diagnosis was confirmed in $67 \%$ of cases by a culture of vertebral biopsy or paraspinal abscess, $20 \%$ by acid fast bacilli (AFB) and conventional PCR tests. The thoracic spine suffering from vertebral collapse was found in most cases and 8 patients had compression of the spinal cord and 5 were left with residual deformities ${ }^{8}$. 
Arcelis and collaborators in 2006, studying ten cases of osteoarticular tuberculosis in children, found that most were affected in T1-T12 of the thoracic spine T1-T12. In six cases complications occurred - kyphosis, they stopped walking and deformities, which are more common in late diagnosis, sometimes made up two years after the onset of symptoms. As demonstrated in the case reported, above (2-16 weeks) as describes the literature is necessary to establish the diagnosis and specific therapy ${ }^{8,9}$.

As early TB test, a positive tuberculin test is strongly indicative of the existence of tuberculosis, but negativity, as observed in this case, and it does not exclude the diagnosis. The NMR is considered the imaging test of choice since it detects early changes that are not seen in conventional radiography or tomography. In addition, it enables soft tissues, neural structures and the paravertebral area to be better detailed ${ }^{10,11}$. In the case described, here changes found in the magnetic resonance imaging (MRI) revealed a severe stage of the disease, probably due to the delay in diagnosis and treatment. The patient received a specific treatment one week after had arrived in the specialist service department.

In the most severe form of Pott's disease, spinal deformation and collapse can compress the spinal cord, thus causing neurological disorders such as paresis and paralyses ${ }^{12}$. In this case report, the patient did not display neurological signs, despite the compressive effects exerted by the posterior displacement in the T11 and T12 vertebral bodies. Unlike adults, who have a higher incidence of neurological symptoms with more localized inflammatory signals, in children, the disease is characterized by an extensive and diffused involvement, the formation of abscess and the low incidence of Pott's paraplegias or tetraplegias ${ }^{10}$. Histological analysis of the lesions, although nonspecific, demonstrated diagnostic value by providing evidence of chronic granulomatous inflammation, as found in most cases ${ }^{9}$.

As this is a paucibacillary form of TB, it remains a challenge to identify the etiologic agent by routine screening. STNPCR is a variant of PCR, which is currently being used, in CPqAM/ FIOCRUZ, and has been giving promising results in noninvasive, clinical samples, collected in outpatient clinics, from patients with paucibacillary $\mathrm{TB}^{6}$. In the case described here, the molecular test, despite being negative for blood, was positive in bone aspirate and urine.

There is still no consensus, based on well-controlled trials, about managing a child with tuberculous spondylitis (Pott's disease). There are controversial issues such as the length of therapy (6 to 12 months, or more), the role of corticosteroids and when surgery should be recommended ${ }^{8}$.
The early detection of disease and referral to a specialist service for rapid diagnostic's investigation are measures that helps on patient prognosis. It should be a concern of pediatricians when they come across chronic cases of back pain and arthralgia in endemic areas.

\section{ACKNOWLEDGMENTS}

The authors are grateful to Dr. Giselly Veríssimo, a physican of the Orthopedics Service, Hospital das Clinicas, Federal University of Pernambuco, for having referred this case for diagnostic investigation. They would also like to thank $\mathrm{CNPq}$ and FACEPE for its financial support and the Centro de Pesquisas Aggeu Magalhães/FIOCRUZ for the use of their equipment and facilities, and technical staff of these two institutions for their assistance.

\section{REFERENCES}

1. Ministério da Saúde. Secretaria de Vigilância Sanitária em Saúde. Tuberculose: casos confirmados notificados no Sistema de Notificação de Agravos de Notificação. Brasília: Ministério de Saúde; 2010.

2. Shikhare SN, Singh DR, Shimpi TR, Peh WC. Tuberculous osteomyelitis and spondylodiscitis. Semin Musculoskelet Radiol 2011; 15:446-458.

3. Medical Research Council Working Party on Tuberculosis of the Spine. Twelfth report of the Medical Research Council Working Party on Tuberculosis of the Spine. Controlled trial of short-course regimens of chemotherapy in the ambulatory treatment of spinal tuberculosis. Results at three years of a study in Korea. J Bone Joint Surg Br 1993; 75:240-248.

4. Schettino LC, Carelli LE, Barbosa MO. Tuberculose vertebral: análise descritiva de uma série de casos submetidos a tratamento cirúrgico. Coluna/Columna 2010; 9:119-125

5. Esposito A, Nascimento LS, Rozov T. Tuberculose de coluna cervical baixa em criança: relato de caso. Pediatria (São Paulo) 2000; 22:172-177.

6. Lima JFC. Detecção do Mycobacterium tuberculosis em amostras de sangue e urina através da Nested-PCR em único tubo [Dissertation]. [Recife]: Centro de Pesquisa Aggeu Magalhães; 2009. 81 p.

7. Cabral MML, Azevedo BCCA, Montenegro LML, Montenegro RA, Lima AS, Schindler HC. Espondilite tuberculosa em adolescente. J Bras Pneumol 2005; 31:261-264.

8. Eisen S, Honywood L, Shingadia D, Novelli V. Spinal tuberculosis in children. Arch Dis Child 2012; 97:724-729.

9. Arcelis RRM, Marques HHS, Matielo RLB, Sakane PT, Baldacci ER.Tuberculose Ostearticular em crianças. Pediatria (São Paulo) 2006; 28:169-174.

10. Moreira, CP; Carvalho, MAP. Tuberculose osteoarticular: apresentação de 38 casos. Rev Bras Reumatol 1980; 20: 251-259.

11. Morris BS, Varma R, Garg A, Awasthi M, Maheshwari M. Multifocal musculoskeletal tuberculosis in children: appearances on computed tomography. Skeletal Radiol 2002; 31:1-8.

12. Jacobs RF, Starke JR. Tuberculosis in children. Med Clin North Am 1993; 77:1335-1351. 